\title{
A Real-World Experience of Mycophenolate Mofetil for Systemic Sclerosis: A Retrospective Multicenter Observational Study
}

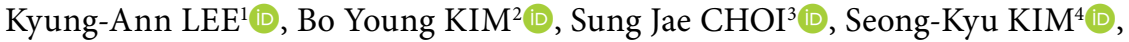 \\ Sang-Hyon $\mathrm{KIM}^{5}{ }^{\mathbb{D}}$, Hyun-Sook $\mathrm{KIM}^{1}{ }^{1}$ \\ ${ }^{1}$ Department of Internal Medicine, Soonchunhyang University School of Medicine, Seoul, South Korea \\ ${ }^{2}$ Department of Internal Medicine, Gangneung Asan Hospital, Gangneung, South Korea \\ ${ }^{3}$ Department of Internal Medicine, Korea University, Ansan, South Korea \\ ${ }^{4}$ Department of Internal Medicine, Catholic University of Daegu School of Medicine, Daegu, South Korea \\ ${ }^{5}$ Department of Internal Medicine, Keimyung University Dongsan Medical Center, Daegu, South Korea
}

\begin{abstract}
Objectives: This study aims to assess the efficacy and safety of mycophenolate mofetil (MMF) on lung function and skin thickness in Korean patients with systemic sclerosis-interstitial lung disease (SSc-ILD) in a real-world setting.

Patients and methods: This retrospective, medical chart-based study was performed at four centers in South Korea and included 34 patients ( 2 males, 32 females; median age 50.5 years; range, 25 to 72 years) with SSc-ILD. We investigated changes in forced vital capacity (FVC), diffusion capacity of the lung for carbon monoxide (DLCO), and modified Rodnan skin score (mRSS) according to MMF treatment for 24 months.

Results: The mean dose and treatment duration of MMF were 1,338.2 $\pm 439.0 \mathrm{mg} /$ day and $13.1 \pm 9.3$ months, respectively. Although FVC decreased significantly at 15 months, FVC and DLCO did not change significantly during treatment with MMF. Median mRSS decreased significantly from $17.5(2-40)$ to $10.5(2-40)$ units $(p<0.0001)$. Thirteen patients $(38.24 \%)$ discontinued treatment with MMF [treatment duration 8.00 (3.0-24.0) months]; the predominant cause of discontinuation was of economic nature (46.15\%). No serious adverse events were reported.

Conclusion: This real-world based study supports the role of MMF in the stabilization of lung function and the improvement in skin thickness with an acceptable safety profile in patients with SSc. Economic burden is the main cause of discontinued treatment with MMF.

Keywords: Interstitial, Korea, lung diseases, mycophenolic acid, scleroderma, systemic.
\end{abstract}

Systemic sclerosis (SSc) is a rare autoimmune disease, which involves multiple organs and often results in significant disability and mortality. SSc-interstitial lung disease (SSc-ILD) is now a leading cause of death in patients with SSc. ${ }^{1,2}$ The 2017 update from the European League Against Rheumatism (EULAR) recommends cyclophosphamide (CYC) and methotrexate as an effective therapeutic option for lung and skin disease caused by SSc. The EULAR recommendations mentioned mycophenolate mofetil (MMF) as a research agenda in order to evaluate the efficacy and safety in the treatment of SSc. ${ }^{3}$ However, a high-quality randomized controlled trial (RCT), which compared MMF (up to $3 \mathrm{~g} /$ day for two years) with oral CYC (2 $\mathrm{mg} / \mathrm{kg} /$ day with a one year follow-up and by matching a placebo for the second year) in

Received: August 21, 2019 Accepted: October 31, 2019 Published online: January 13, 2020

Correspondence: Hyun-Sook Kim, MD. Division of Rheumatology, Department of Internal Medicine, Soonchunhyang University Seoul Hospital, 59 Daesagwan-ro, Yongsan-gu, 04401 Seoul, Republic of Korea. Tel: +82-2-710-3063 e-mail: healthyra@schmc.ac.kr

\section{Citation:}

Lee KA, Kim BY, Choi SJ, Kim SK, Kim SH, Kim HS. A Real-World Experience of Mycophenolate Mofetil for Systemic Sclerosis: A Retrospective Multicenter Observational Study. Arch Rheumatol 2020;35(3):366-375. 
patients with SSc-related ILD, ${ }^{4}$ caused a move as a first-line preference for ILD induction therapy from CYC to MMF among experts. ${ }^{5}$ MMF versus oral CYC in scleroderma-related interstitial lung disease (SLS II) study showed that forced vital capacity (FVC) significantly improved in both MMF- and CYC-treated groups and MMF had a superior safety profile. ${ }^{4}$ SLS II trials also demonstrated significant improvements in the total modified Rodnan skin score (mRSS) using MMF.

Although RCTs represent the gold-standard for medical evidence to assess drug efficacy, this strict set of criteria and fixed patterns of treatment could affect the generalizability of results. Compared with RCTs, observational studies based on real-world data had numerous potential confounders; however, they had more of a practical nature rather than a tightly controlled design. Real-world data can also provide unrevealed treatment characteristics and safety profiles, which have not been captured by RCTs. ${ }^{6-8}$ A retrospective observational study showed that a treatment with lower than $3 \mathrm{~g}$ a day of MMF (majority of patients were on $2 \mathrm{~g} /$ day) slowed the rate of decline in lung function in patients treated with SSc-ILD. An Australian scleroderma cohort study observed that MMF (mean dose of $1.9 \pm 0.4 \mathrm{~g} /$ day and mean duration of $2.7 \pm 1.7$ years) was associated with improvements in mRSS and showed good tolerability. However, studies conducted for Asian patients with $\mathrm{SSc}$ on the efficacy and safety of MMF are insufficient. Particularly in Korea, real-world based observational studies are lacking, due to the fact that the use of MMF for SSc-ILD has been limited in clinical practice as there is limited coverage by Korean national health insurance. Therefore, in this study, we aimed to assess the efficacy and safety of MMF on lung function and skin thickness in Korean patients with SSc-ILD in a real-world setting.

\section{PATIENTS AND METHODS}

This retrospective, medical chart-based research was performed at four centers in South Korea (Soonchunhyang University Seoul hospital, Korea University medical center, Catholic University Daegu medical center, and Keimyung University Dongsan medical center) between March 2014 and March 2017 and included 34
SSc-ILD patients ( 2 males, 32 females; median age 50.0 years; range, 25 to 72 years). The inclusion criteria were $\mathrm{SSc}$ with the use of MMF having either progressive lung disease or inadequate responses with other treatments between March 2014 and March 2017. All patients fulfilled the 2013 EULAR/American College of Rheumatology classification criteria for SSc. ${ }^{9}$ Patients were excluded if (i) they were over 75 years old or under 20 years old; (ii) had previous immunosuppressant treatment for $>4$ months or use of any immunosuppressant drug other than MMF or CYC within the month prior to study entry. The study protocol was approved by the Institutional Review Board for Human Research of each center (SCH 2015-001). A written informed consent was obtained from each patient. The study was conducted in accordance with the principles of the Declaration of Helsinki.

The primary endpoint was the changes in lung functions over time. Lung function was assessed by FVC (FVC: \% predicted) and diffusion capacity of the lung for carbon monoxide (DLCO: \% predicted) (corrected for hemoglobin). The secondary endpoints were changes in skin thickness scores obtained via the mRSS, ${ }^{10}$ semiquantitative scores of nailfold capillaroscopy (NFC), and safety profile. NFC scores of megacapillary, avascular area, and ramification were used, with scores ranging from 0 to 3 ; where $0=$ no change, $1=$ less than $33 \%$ capillary alteration/reduction, $2=33 \%-66 \%$ of capillary alteration/reduction, $3=$ greater than $66 \%$ capillary alteration/reduction, per linear mm. ${ }^{11-13}$ The basic demographic information, medication use, and presence of organ involvement of SSc were also obtained for all patients. The repetitive clinical assessment, including spirometry, DLCO (corrected for hemoglobin), mRSS, and semiquantitative scores of NFC were acquired at 3, 9, 15 , and 24 months.

\section{Statistical analysis}

All statistical analyses were performed using the PASW for Windows version 17.0 software (SPSS Inc., Chicago, IL, USA). Demographic and baseline data were analyzed using the Mann-Whitney U test. Categorical variables were compared between groups with the Chi-square test and Fisher's exact test. The Wilcoxon signed rank 


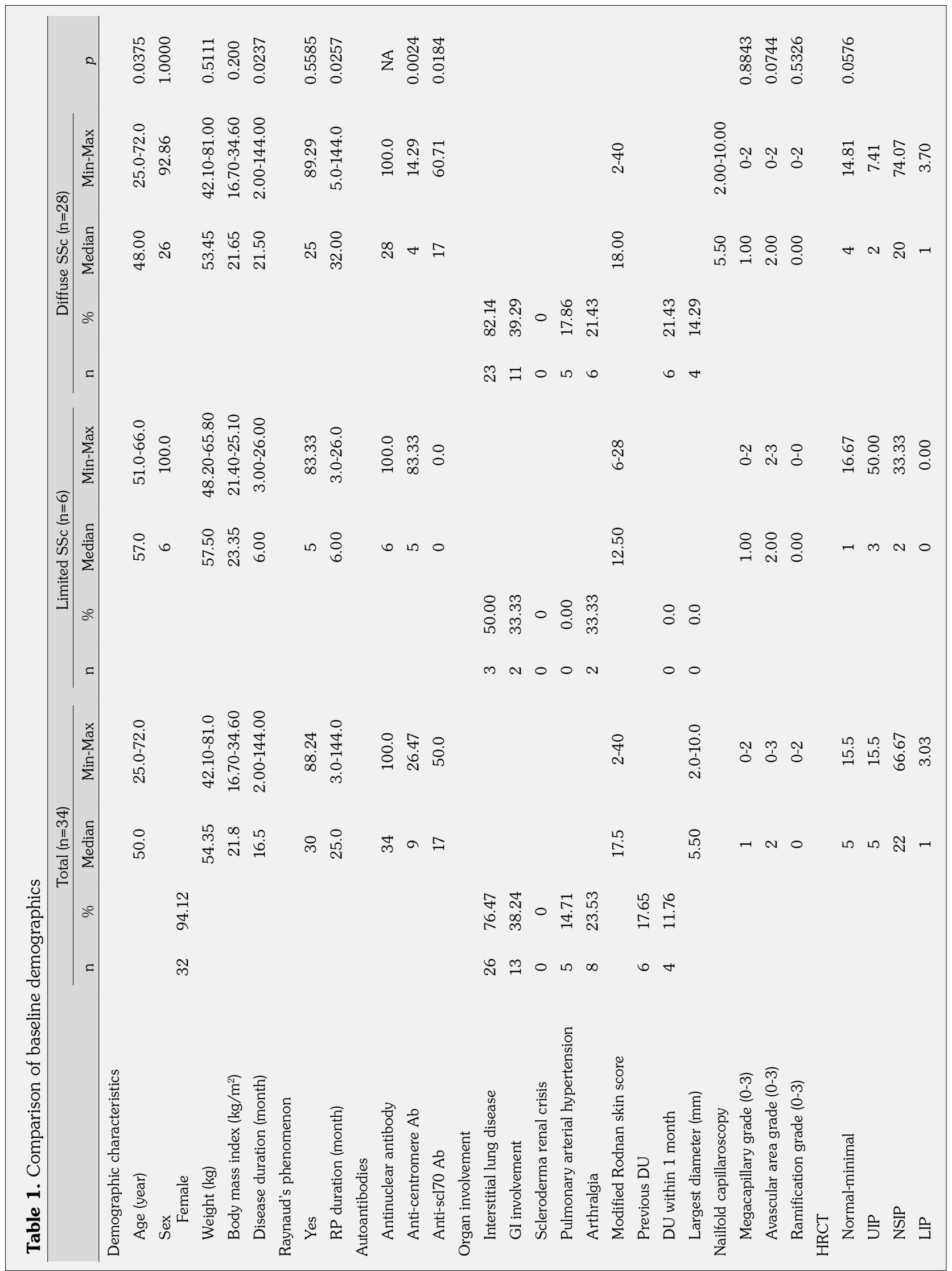




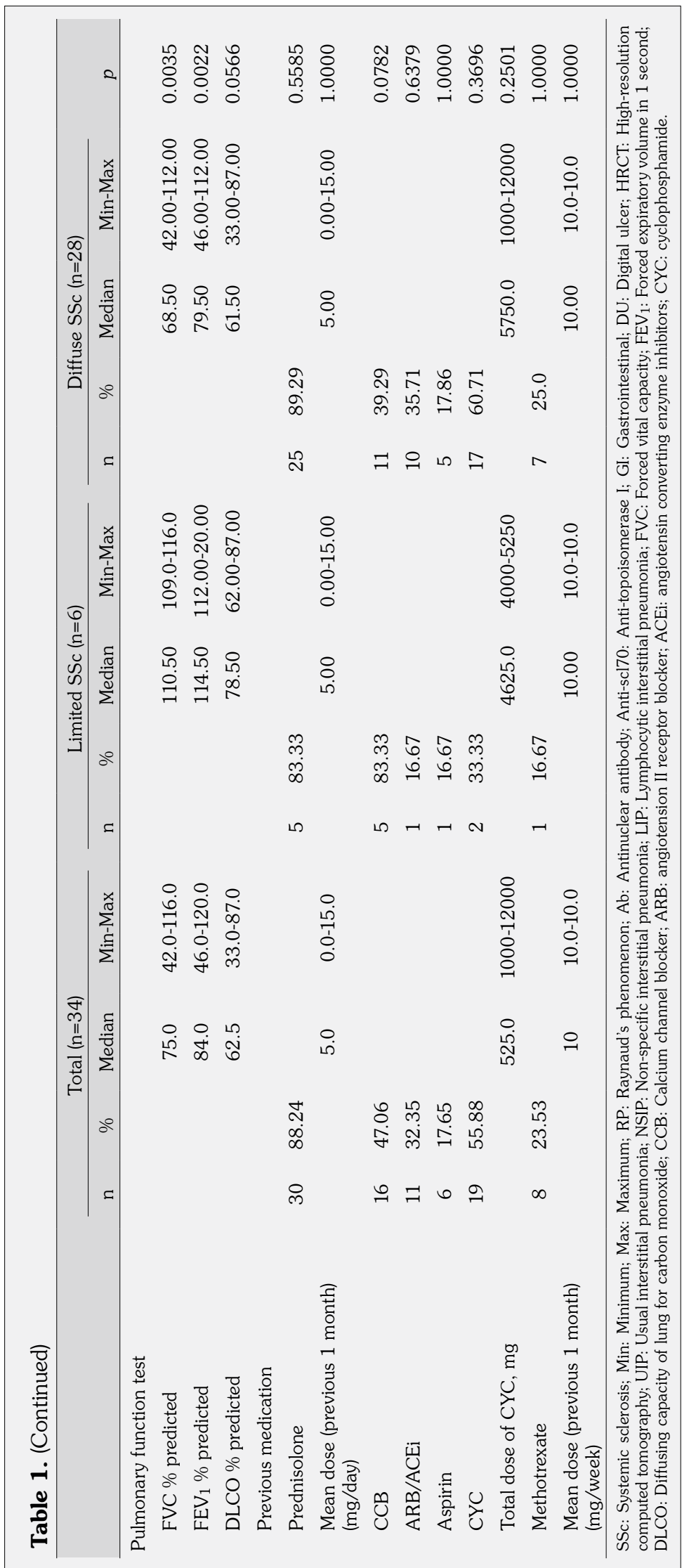


test was used to assess the effect of MMF on FVC, DLCO, and mRSS. Spearman's rank correlation was used to assess correlations between treatment duration and clinical parameters. A $p$ value $<0.05$ was considered statistically significant.

\section{RESULTS}

A total of 34 patients with SSc were enrolled including 28 patients with diffuse cutaneous $\mathrm{SSc}$ and six patients with limited cutaneous $\mathrm{SSc}$. The baseline characteristics of the study population are shown in Table 1. Patients' median FVC and DLCO were 75.0 (42.0-116.0) \%, 62.5 (33.0-87.0) \%, respectively. A comparison of demographic features between limited and diffuse SSc showed that patients with diffuse cutaneous SSc were younger, had a longer duration of disease period, higher positivity of anti-topoisomerase I, and lower FVC than patients with limited cutaneous SSc. There was no significant difference in presence of organ involvement, semi-quantitative scores of NFC, initial mRSS, DLCO, and use of medications.

Median FVC (median [min-max]) was 75.0 (42.0-116.0) \% ( $n=28), 75.0(42.0-113.0) \%(n=15)$, $72.5(46.0-110.0) \%(\mathrm{n}=14), 68.5(46.0-103.0) \%$ $(\mathrm{n}=10)$, and $60.5(47.0-96.0) \%(\mathrm{n}=4)$ at baseline, 3, 9, 15, and 24 months, respectively. Median FVC was significantly decreased at 15 months $(p=0.028)$, otherwise, FVC was not significantly changed (Figure 1a).

Median DLCO was 62.5 (min-max 33.0-87.0) \% ( $\mathrm{n}=28), 65.5$ (30.0-102.0) $\% \quad(n=15), \quad 66.0 \quad(38.0-82.0) \quad \% \quad(n=14), 69.0$ (40.0-77.0) \% ( $n=10)$, and 58.5 (34.0-78.0) \% $(n=4)$ at baseline, 3, 9, 15, and 24 months, respectively. Median DLCO was not significantly changed during treatment with MMF (Figure 1b). The DLCO and the last dose of MMF showed a positive correlation; however, it was insignificant $(\mathrm{r}=0.255$, $p=0.279$ ). The patients were stratified into three groups by the severity of DLCO abnormality at baseline: mild (60-79\%), moderate (40-59\%), and severe decrease (below 40\%). We then analyzed the effect of MMF according to the severity of DLCO at baseline. The median DLCO was not statistically different in the stratification of DLCO severity (data not shown).

The mean dose of MMF was $1338.2 \pm 439.0 \mathrm{mg} /$ day, and mean treatment duration was $13.1 \pm 9.3$ months. Median dose of MMF (median [min-max]) at baseline, 3, 9, 15, and 24 months was 1,250 (500-2,000) mg/day $(\mathrm{n}=34), \quad 1,250 \quad(500-2,000) \mathrm{mg} / \mathrm{day} \quad(\mathrm{n}=34)$, $1,500 \quad(500-2,000) \mathrm{mg} /$ day $(\mathrm{n}=19), \quad 1,250$ $(500-2,000) \mathrm{mg} / \mathrm{day}(\mathrm{n}=16)$, and 1,000 $(1,000-2,000) \mathrm{mg} /$ day $(\mathrm{n}=6)$, respectively. The dosage of MMF tended to increase according to the treatment duration; however, without (a)

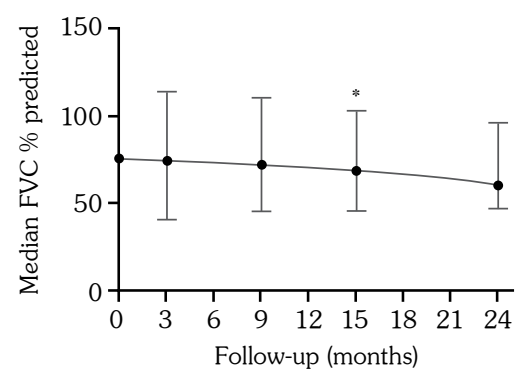

(b)

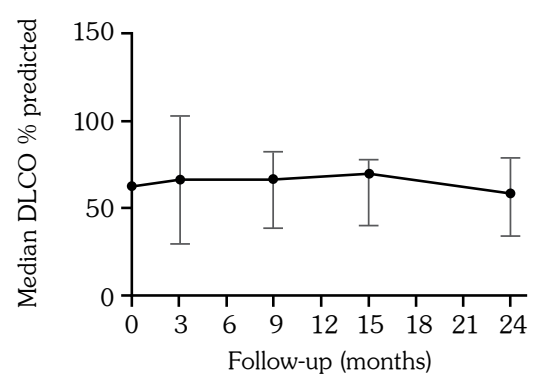

(c)

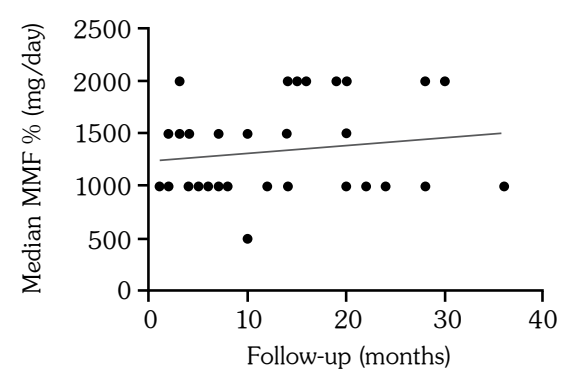

Figure 1. (a) Changes on the \% predicted forced vital capacity (FVC). The number of patients was 28 at baseline, 15 at 3 months, 14 at 9 months, 10 at 15 months, and 4 at 24 months. The vertical line represent the range. Median FVC was significantly decreased at 15 months $(p=0.028)$, otherwise, FVC was not significantly changed. (b) Changes on \% predicted diffusion capacity of lung for carbon monoxide. Median DLCO was not significantly changed during treatment with MMF. (c) Changes on dosage of MMF. The last dosage of MMF tends to increase according to treatment duration without statistical significance $(r=0.128, p=0.469)$.

${ }^{*} \mathrm{p}<0.05$; FVC: Forced vital capacity; DLCO: Diffusion capacity of lung for carbon monoxide; MMF: Mycophenolate mofetil. 
(a)

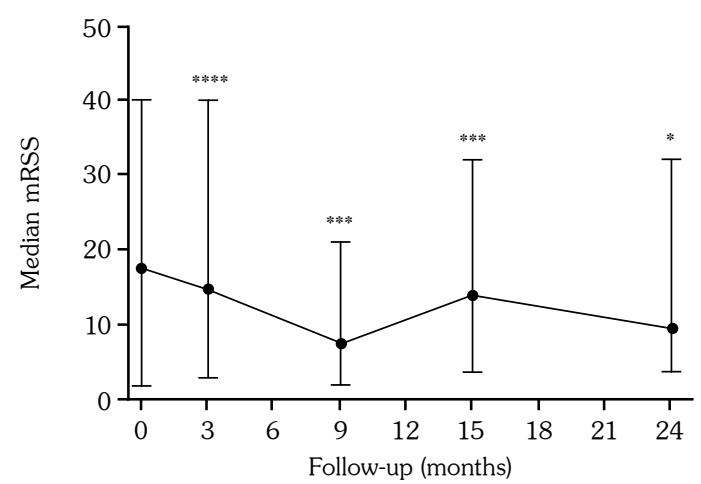

(b)

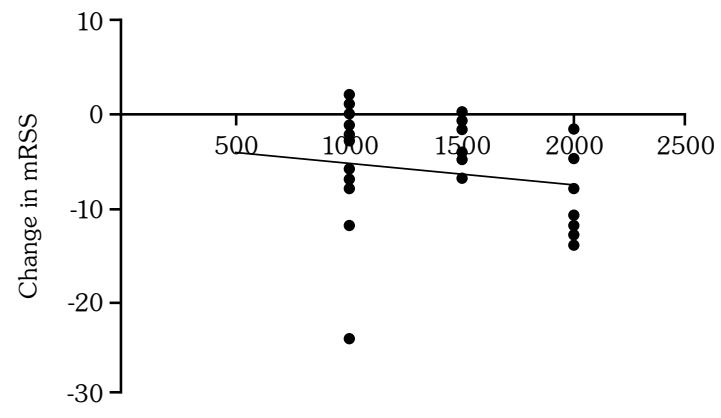

Last dose of MMF (mg/day)

Figure 2. (a) Changes of the modified Rodnan total skin score over 24 months. The number of patients was 34 at baseline, 29 at 3 months, 15 at 9 months, 13 at 15 months, and 6 at 24 months. The vertical line represent the range. The mRSS significantly decreased after beginning of the treatment with MMF. (b) Last dose of mycophenolate mofetil and changes in modified Rodnan skin score shows negative correlation without statistical significance $(\mathrm{r}=-0.202, \mathrm{p}=0.251)$.

mRSS: Modified Rodnan skin score; MMF: Mycophenolate mofetil; " $\mathrm{p}<0.05,{ }^{* * * *} \mathrm{p}<0.001,{ }^{* * * * *} \mathrm{p}<0.0001$.

statistical significance $(\mathrm{r}=0.128, \mathrm{p}=0.469)$ (Figure 1c). On the other hand, there were no significant changes in the dose of MMF, and the last dose of steroids was significantly decreased from $5.0(0-15.0) \mathrm{mg} /$ day to $2.5(0-15) \mathrm{mg} /$ day $(p=0.007)$. The use of calcium channel blocker, angiotensin converting enzyme inhibitors/ angiotensin II receptor blocker, and aspirin did not significantly change overtime after treatment with MMF.

The median mRSS (median [min-max]) was significantly improved after treatment with MMF from $17.5(2-40)$ units to $10.5(2-40)$ units $(\mathrm{p}<0.0001)$. The median mRSS was $15.0(2-40)$ units $(n=29), 8.0(3-40)$ units $(n=15), 14(4-32)$ units $(n=13), 9.5(4-32)$ units $(n=6)$ at $3,9,15$, and 24 months, respectively. Using observational data, there was a statistically significant improvement in
mRSS at 3, 9, 15, and 24 months compared to baseline $(p<0.001, p=0.002,0.002$, and 0.027 , respectively) (Figure 2a). The correlation between dose of MMF and changes of mRSS was not significant $(r=-0.202, p=251)$ (Figure 2b).

Treatment with MMF did not show any significant changes in semi-quantitative scores of mega-capillary, avascular area, or ramification grades of NFC overtime (Table 2).

A total of 13 patients (38.24\%) discontinued treatment with MMF, the predominant reason of discontinuation was due to economic challenges $(\mathrm{n}=6,46.15 \%)$. Other causes included inadequate responses to MMF $(n=2)$, death $(n=1)$, missed follow-ups $(n=1)$, inefficacy $(n=2)$, and pregnancy plans $(\mathrm{n}=1)$ (Table 3). The median treatment duration in patients who discontinued MMF was 8.00 (3.0-24.0) months. The non-lethal adverse

Table 2. Semi-quantitative scores of nailfold capillaroscopy at baseline and after treatment with mycophenolate mofetil

\begin{tabular}{|c|c|c|c|c|c|c|c|c|c|c|c|}
\hline \multirow[b]{2}{*}{$\begin{array}{l}\text { Nailfold capillaroscopic } \\
\text { scores }\end{array}$} & \multicolumn{2}{|c|}{ Initial $(n=24)$} & \multicolumn{2}{|c|}{3 months $(n=4)$} & \multicolumn{2}{|c|}{9 months $(\mathrm{n}=9)$} & \multicolumn{2}{|c|}{15 months $(n=4)$} & \multicolumn{2}{|c|}{24 months ( $\mathrm{n}=2$ ) } & \multirow[b]{2}{*}{$p$} \\
\hline & Median & Min-Max & Median & Min-Max & Median & Min-Max & Median & Min-Max & Median & Min-Max & \\
\hline Maga-capillary (0-3) & 1 & $0-2$ & 0 & $0-1$ & 1 & $0-1$ & 0.5 & $0-2$ & 0.5 & $0-1$ & 0.125 \\
\hline Avascular area (0-3) & 2 & $0-3$ & 1.5 & $1-3$ & 1 & $3-1$ & 2 & $1-2$ & 2.5 & $1-3$ & 0.531 \\
\hline Ramification (0-3) & 0 & $0-2$ & 0 & $0-0$ & 0 & $0-3$ & 0 & $0-0$ & 0 & $0-0$ & 0.500 \\
\hline
\end{tabular}


Table 3. Treatment duration and cause of discontinuation

\begin{tabular}{lccccc}
\hline & $\mathrm{n}$ & $\%$ & Mean \pm SD & Median & Range \\
\hline Overall treatment duration & 34 & & $13.1 \pm 9.3$ & 12.00 & $3.0-36.0$ \\
Treatment duration in discontinued group & 13 & & $10.9 \pm 7.7$ & 8.00 & $3.0-24.0$ \\
Maximum dose of discontinued group & 13 & & $1,346.2 \pm 375.5$ & 1,500 & $1,000-2,000$ \\
Cause of discontinuation & & & & & \\
Economic problem & 6 & 46.15 & & \\
Adequate response & 2 & 15.38 & & \\
Inefficacy & 2 & 15.38 & & \\
Death & 1 & 7.69 & & \\
Follow-up loss & 1 & 7.69 & & \\
Adverse events & 0 & 0 & \\
$\quad$ Prepare pregnancy & 1 & 7.69 & \\
SD: Standard deviation. & & &
\end{tabular}

events included leukopenia $(n=2)$, diarrhea $(n=2)$, and minor infection $(n=3)$. One death occurred due to acute exacerbation of SSc-ILD.

\section{DISCUSSION}

The present real-world based observational retrospective study assessed the effect of MMF on treatment and safety of SSc in Korea. The results of this study have shown that treatment with MMF is associated with a reduction in the extent of cutaneous sclerosis; however, not with improvements in lung function tests. We have also confirmed the acceptable safety profile of MMF. Considering that there is a progressive decrease in lung function in SSc-related ILD, which is the natural course of SSc-related ILD, ${ }^{14}$ non-significant decrease of DLCO in the current study supports the beneficial effects of MMF on stabilization of lung function.

Compared to previous studies which reported improvements of lung function, the analysis of this real-world based data has demonstrated disappointing results by particularly showing significant decrease of FVC at 15-month MMF treatment. The possible explanation of these findings could include relatively lower dose of MMF (mean dose 1338.2 $\pm 439.0 \mathrm{mg} /$ day) and short treatment duration (mean treatment duration $13.1 \pm 9.3$ months) compared to previous studies. The SLS II study, which is a head-to-head comparative study of MMF versus oral CYC, has led to the change of first-line preference for SSc-related ILD from CYC to MMF, according to the expert opinion based treatment algorithms for SSc. ${ }^{5}$ In the SLS II study, the patients who completed the entire treatment of MMF showed an overall improvement of FVC, whereas the premature withdrawal population from the treatment had an overall worse FVC (75\%). Moreover, most patients in the MMF group reached and maintained the target dose (3 g/day). ${ }^{4}$ In other reported studies, pulmonary function has also improved or stabilized after treatment with at least more than $2 \mathrm{~g} /$ day of MMF. ${ }^{15-18}$ The SLS II study has shown an average peak improvement of FVC at 18-21 months. The Australian Scleroderma Cohort study has demonstrated that treatment with MMF was associated with stability of the SSc-ILD for up to 36 months. ${ }^{19}$ Results from our study and previous data suggest that optimal dosage of MMF for an adequate period of time is essential to prevent progression of SSc-ILD.

Interstitial lung disease accounts for up to $30-35 \%$ of mortality in patients with SSc. ${ }^{20}$ The standard treatments for ILD includes MMF and CYC, both of which have shown improved pulmonary function. ${ }^{1}$ Although there are numerous positive results of MMF treatment in the attenuation of the disease, ${ }^{4,18,21}$ MMF for SSc-related ILD is not covered by the Korean national health insurance. Owing to the high burden of expenses of this drug, the treatment options of SSc-related ILDs are definitely limited. High costs of MMF 
could lead to suboptimal dosage and duration of the treatment in our study. Based on our realworld experience, the financial support through expansion of reimbursement guidelines is urgently needed to provide appropriate treatment with MMF with sufficient dose and period in patients with SSc.

A substantial number of studies have highlighted the therapeutic effectiveness of MMF on skin involvement in patients with SSc. ${ }^{4,16,22-26}$ In the current study, we have also observed significant decline in mRSS associated with MMF treatment. The baseline mRSS in the present study versus SLS II was similar (17.5 [range 2-40] in the current study versus $15.3 \pm 10.4$ in SLS II). Although the dosage of MMF was lower in our study than in SLS II trials, the observed changes of mRSS at 24 months from baseline were comparable (-7.0 in the current study versus -4.9 in SLS II). ${ }^{4,22}$ A prospective, observational cohort study has also shown that $2 \mathrm{~g}$ of MMF was associated with significant reductions in mRSS at 12 months (-4.1 units) in patients with early diffuse cutaneous SSc. ${ }^{27}$ Although CYC and hematopoietic stem cell transplantation have demonstrated clear benefits for skin in $\mathrm{SSc},{ }^{28}$ the high risk limits the use of those treatments, and alternative safe options are necessary in clinical practice. For treatment of skin involvement, MMF lower than $2 \mathrm{~g}$ /day may be sufficient. Further studies are needed to define the proper dosage of MMF according to organ-based complications, considering the risks and benefits. Accumulative evidence of consistent effects may lead to incorporation of MMF as the preferred treatment option for skin manifestations in patients with SSc in the near future.

Although treatment of the underlying disease with a suitable immunosuppressant is important, the role of immunosuppressive therapy is unclear in patients with autoimmune diseases associated with Raynaud's phenomenon. ${ }^{29}$ Additionally, there have been no studies evaluating the effects of MMF on microcirculation in SSc. We have investigated the changes of semi-quantitative scores of NFC at 24 months after MMF treatment in patients with SSc; however, we failed to show any significant improvement of the microvasculature structure. MMF administration was reported to restore renal microvascular oxygenation, reactive oxygen species (ROS) production, and immune cell-mediated inflammatory activation in a rat model of supra-renal aortic occlusion-mediated ischemia/reperfusion injury. ${ }^{30}$ Although inflammatory cascade and ROS play a role in the pathogenesis of $\mathrm{SSc},{ }^{31}$ structural and functional abnormalities of microvasculature are the main drivers for digital ischemia. ${ }^{32}$ Therefore, immunosuppressants including MMF could be ineffective to obliterative vasculopathy in patients with SSc.

We have confirmed an acceptable safety of MMF in patients with SSc. In the present study, leukopenia, diarrhea, and minor infections were observed in $5.9 \%, 5.9 \%$, and $2.9 \%$ of the patients, respectively. Likewise, leukopenia and pneumonia occurred in $5.7 \%$ and $10.1 \%$ of MMF-treated patients in SLS II study. ${ }^{4}$ No serious adverse events related to MMF treatment were observed.

The main weakness of this study is its observational, retrospective, and uncontrolled design with a relatively small number of patients. Moreover, treatment with MMF was not compared to the use of placebo or other immunosuppressive agents such as CYC. About $40 \%$ of enrolled patients $(n=13)$ discontinued treatment with MMF (mean treatment duration of less than one year), only six patients retained the treatment with MMF at 24 months (the remaining 15 patients on MMF treatment did not reach 24 months), and the data about changes on lung function and skin thickening were limited. We suggest that suboptimal dosage and treatment duration of MMF may cause a significant decline of FVC and a non-significant decrease of DLCO. Therefore, to confirm our speculation, further studies on the efficacy of MMF according to the dosage and duration are needed. Furthermore, this is a multicenter observational study, thus the inter-observer reliability of mRSS was not studied, which is another limitation of our study. However, the same experienced examiners in each institution have evaluated skin thickness throughout the trial, and good inter-observer reliability was observed in previous studies of mRSS. ${ }^{33}$

In conclusion, our observational study supports the role of MMF in the stabilization of DLCO and FVC, and the improvement in 
skin thickness in patients with SSc. However, meaningful improvement of lung function was not achieved with suboptimal dosage and duration of MMF in a real-world setting. Despite the favorable safety profile of MMF, economic burden is the main cause of discontinued treatment with MMF.

\section{Declaration of conflicting interests}

The authors declared no conflicts of interest with respect to the authorship and/or publication of this article.

\section{Funding}

This study is supported by fund of the Soonchunhyang University.

\section{REFERENCES}

1. Denton CP, Khanna D. Systemic sclerosis. Lancet 2017;390:1685-99.

2. Koo SM, Uh ST. Treatment of connective tissue disease-associated interstitial lung disease: the pulmonologist's point of view. Korean J Intern Med 2017;32:600-10.

3. Kowal-Bielecka O, Fransen J, Avouac J, Becker M, Kulak A, Allanore Y, et al. Update of EULAR recommendations for the treatment of systemic sclerosis. Ann Rheum Dis 2017;76:1327-39.

4. Tashkin DP, Roth MD, Clements PJ, Furst DE, Khanna D, Kleerup EC, et al. Mycophenolate mofetil versus oral cyclophosphamide in sclerodermarelated interstitial lung disease (SLS II): a randomised controlled, double-blind, parallel group trial. Lancet Respir Med 2016;4:708-19.

5. Fernández-Codina A, Walker KM, Pope JE. Scleroderma algorithm group. treatment algorithms for systemic sclerosis according to experts. Arthritis Rheumatol 2018;70:1820-8.

6. Jung UH, Kwak SG, Choe JY, Lee SS, Kim SK. The Effect of Mycophenolate Mofetil on Non-Renal Manifestations in Systemic Lupus Erythematosus: Results from Korean Lupus Network Registry. J Korean Med Sci 2019;34:e185.

7. Kim HS, Lee S, Kim JH. Real-world evidence versus randomized controlled trial: clinical research based on electronic medical records. J Korean Med Sci 2018;33:e213.

8. Monti S, Grosso V, Todoerti M, Caporali R. Randomized controlled trials and real-world data: differences and similarities to untangle literature data. Rheumatology (Oxford) 2018;57(57 Suppl 7):vii54-vii58.

9. van den Hoogen F, Khanna D, Fransen J, Johnson SR, Baron M, Tyndall A, et al. 2013 classification criteria for systemic sclerosis: an American College of Rheumatology/European League against Rheumatism collaborative initiative. Arthritis Rheum 2013;65:2737-47.

10. Clements P, Lachenbruch P, Siebold J, White B, Weiner S, Martin R, et al. Inter and intraobserver variability of total skin thickness score (modified Rodnan TSS) in systemic sclerosis. J Rheumatol 1995;22:1281-5.

11. Sulli A, Secchi ME, Pizzorni C, Cutolo M. Scoring the nailfold microvascular changes during the capillaroscopic analysis in systemic sclerosis patients. Ann Rheum Dis 2008;67:885-7.

12. Park SJ, Choi IH, Kim HS. Rapid changes in capillary morphology and architecture in a patient with dermatomyositis. Korean $J$ Intern Med 2019;34:680-2.

13. Ruaro B, Smith V, Sulli A, Decuman S, Pizzorni $\mathrm{C}$, Cutolo M. Methods for the morphological and functional evaluation of microvascular damage in systemic sclerosis. Korean J Intern Med 2015;30:1-5.

14. Morgan C, Knight C, Lunt M, Black CM, Silman AJ. Predictors of end stage lung disease in a cohort of patients with scleroderma. Ann Rheum Dis 2003;62:146-50.

15. Liossis SN, Bounas A, Andonopoulos AP. Mycophenolate mofetil as first-line treatment improves clinically evident early scleroderma lung disease. Rheumatology (Oxford) 2006;45:1005-8.

16. Vanthuyne M, Blockmans D, Westhovens R, Roufosse $\mathrm{F}$, Cogan $\mathrm{E}$, Coche $\mathrm{E}$, et al. A pilot study of mycophenolate mofetil combined to intravenous methylprednisolone pulses and oral low-dose glucocorticoids in severe early systemic sclerosis. Clin Exp Rheumatol 2007;25:287-92.

17. Koutroumpas A, Ziogas A, Alexiou I, Barouta G, Sakkas LI. Mycophenolate mofetil in systemic sclerosisassociated interstitial lung disease. Clin Rheumatol 2010;29:1167-8.

18. Shenoy PD, Bavaliya M, Sashidharan S, Nalianda K, Sreenath S. Cyclophosphamide versus mycophenolate mofetil in scleroderma interstitial lung disease (SScILD) as induction therapy: a single-centre, retrospective analysis. Arthritis Res Ther 2016;18:123.

19. Owen C, Ngian GS, Elford K, Moore O, Stevens W, Nikpour M, et al. Mycophenolate mofetil is an effective and safe option for the management of systemic sclerosis-associated interstitial lung disease: results from the Australian Scleroderma Cohort Study. Clin Exp Rheumatol 2016;34 Suppl 100:170-6.

20. Roofeh D, Jaafar S, Vummidi D, Khanna D. Management of systemic sclerosis-associated interstitial lung disease. Curr Opin Rheumatol 2019;31:241-9.

21. Omair MA, Alahmadi A, Johnson SR. Safety and effectiveness of mycophenolate in systemic sclerosis. A systematic review. PLoS One 2015;10:e0124205.

22. Namas R, Tashkin DP, Furst DE, Wilhalme H, Tseng $\mathrm{CH}$, Roth $\mathrm{MD}$, et al. Efficacy of mycophenolate mofetil and oral cyclophosphamide on skin thickness: 
post hoc analyses from two randomized placebocontrolled trials. Arthritis Care Res (Hoboken) 2018;70:439-44.

23. Le EN, Wigley FM, Shah AA, Boin F, Hummers LK. Long-term experience of mycophenolate mofetil for treatment of diffuse cutaneous systemic sclerosis. Ann Rheum Dis 2011;70:1104-7.

24. Nihtyanova SI, Brough GM, Black CM, Denton CP. Mycophenolate mofetil in diffuse cutaneous systemic sclerosis--a retrospective analysis. Rheumatology (Oxford) 2007;46:442-5.

25. Mendoza FA, Nagle SJ, Lee JB, Jimenez SA. A prospective observational study of mycophenolate mofetil treatment in progressive diffuse cutaneous systemic sclerosis of recent onset. $\mathrm{J}$ Rheumatol 2012;39:1241-7.

26. Derk CT, Grace E, Shenin M, Naik M, Schulz S, Xiong $\mathrm{W}$. A prospective open-label study of mycophenolate mofetil for the treatment of diffuse systemic sclerosis. Rheumatology (Oxford) 2009;48:1595-9.

27. Herrick AL, Pan X, Peytrignet S, Lunt M, Hesselstrand $\mathrm{R}$, Mouthon $\mathrm{L}$, et al. Treatment outcome in early diffuse cutaneous systemic sclerosis: the European Scleroderma Observational Study (ESOS). Ann Rheum Dis 2017;76:1207-18.
28. Sullivan KM, Goldmuntz EA, Keyes-Elstein L, McSweeney PA, Pinckney A, Welch B, et al. Myeloablative Autologous Stem-Cell Transplantation for Severe Scleroderma. N Engl J Med 2018;378:35-47.

29. Wigley FM, Flavahan NA. Raynaud's Phenomenon. N Engl J Med 2016;375:556-65.

30. Ergin B, Heger M, Kandil A, Demirci-Tansel C, Ince $\mathrm{C}$. Mycophenolate mofetil improves renal haemodynamics, microvascular oxygenation, and inflammation in a rat model of supra-renal aortic clamping-mediated renal ischaemia reperfusion injury. Clin Exp Pharmacol Physiol 2017;44:294-304.

31. Vona R, Giovannetti A, Gambardella L, Malorni $\mathrm{W}$, Pietraforte D, Straface E. Oxidative stress in the pathogenesis of systemic scleroderma: An overview. J Cell Mol Med 2018;22:3308-14.

32. Herrick AL. Evidence-based management of Raynaud's phenomenon. Ther Adv Musculoskelet Dis 2017;9:317-29.

33. Khanna D, Furst DE, Clements PJ, Allanore Y, Baron M, Czirjak L, et al. Standardization of the modified Rodnan skin score for use in clinical trials of systemic sclerosis. J Scleroderma Relat Disord 2017;2:11-8. 